\title{
REGULADORES VEGETAIS E O DESBASTE QUÍMICO DE FRUTOS DE TANGOR MURCOTE
}

\author{
Marcio Christian Serpa Domingues*; Elizabeth Orika Ono; João Domingos Rodrigues
}

Depto. de Botânica - IB/UNESP, C.P. 502 - CEP: 18618-000 - Botucatu, SP.

*Autor correspondente <sdomingues@hotmail.com.br>

RESUMO: O tangor 'Murcote' apresenta necessidade de desbaste de frutos devido a alternância de produção, caracterizado por anos de excessiva produção intercalado com anos de baixa produção, evitando assim, a diminuição da qualidade dos frutos. O presente trabalho teve por objetivo avaliar a eficiência de reguladores vegetais, a auxina ANA (ácido naftalenacético) e ethephon (etileno) no desbaste químico de frutos de tangor 'Murcote' aplicado 40 dias após o pleno florescimento. O experimento foi conduzido em Pratânia, SP, onde plantas de 5 anos de idade, enxertadas sobre o limoeiro 'Cravo', foram pulverizadas com ANA a 0, 100, 200, 300 e $400 \mathrm{mg} \mathrm{L}^{-1}$ e com ethephon a 200, 300 e $400 \mathrm{mg} \mathrm{L}^{-1}$, ambos em solução aquosa juntamente com adjuvante não iônico a 0,05\%. A contagem dos frutos foi realizada previamente aos tratamentos em 2 ramos marcados por planta. O ANA não interferiu significativamente no desbaste de frutos, com porcentagens de queda variando entre 7 a 14\%, enquanto que as pulverizações com ethephon mostraram maior eficiência no desbaste de frutos, principalmente na dose de $400 \mathrm{mg} \mathrm{L}^{-1}$, promovendo $66,6 \%$ de queda de frutos, sem contudo induzir a abscisão foliar. As doses inferiores de ethephon também promoveram desbaste de frutos da ordem de $40 \%$. As porcentagens de queda de frutos foram pequenas, para plantas pulverizadas com ANA, enquanto que a aplicação de ethephon promoveu maior eficiência no desbaste de frutos.

Palavras-chave: Citrus reticulata, raleio, ethephon, ácido naftaleno acético

\section{PLANT GROWTH REGULATORS AND 'HONEY' ORANGE FRUIT CHEMICAL THINNING}

\begin{abstract}
Alternate bearing is an important caracteristic of some mandarins that reduces fruit quality and yield in tangor Murcott, and to reduce this effect is necessary to perform fruit thinning to avoid high and low production year by year. To evaluate the effects of plant growth regulators on fruit thinning of the 'honey' orange, an auxin (NAA-naphthalene acetic acid) and ethephon (ethylene), were applied 40 days after full bloom in an experiment carried out at Pratania, S.P., Brazil. Five-year-old plants grafted on Rangpur lime were used, sprayed with NAA $\left(0,100,200,300\right.$ e $400 \mathrm{mg} \mathrm{L}^{-1}$ and ethephon $\left(200,300,400 \mathrm{mg} \mathrm{L}^{-1}\right)$, both in water solution, containing $0.05 \%$ of non ionic wetting agent. Fruit number was determined before treatment applications in two shoots per plant. NAA did not promote significant differences on fruit thinning (between 7 to $14 \%$ ), however, the application of ethephon, at all rates, increased fruit thinning by 41.6 to $66.6 \%$, with concentrations of 200 to $400 \mathrm{mg} \mathrm{L}^{-1}$ respectively, without leaf drop. Application of NAA did not promote chemical fruit thinning whereas ethephon showed high percentage of fruit thinning after full bloom with increased concentrations.
\end{abstract}

Key words: Citrus reticulata, thinning, ethephon, naphthalene acetic acid

\section{INTRODUÇÃO}

O Brasil é o quarto maior produtor mundial de tangerinas, incluindo o híbrido tangor 'Murcote', com produção de $762.000 \mathrm{t}$ de um total de $13.323 .000 \mathrm{t}$ colhidas em todo o mundo. São Paulo detém $46 \%$ da produção nacional, seguido do Rio Grande do Sul que produz 18\%, Paraná produz $13 \%$ e Minas Gerais responde por 6\% (Agrianual, 2000) .

A maioria das variedades cítricas comerciais não requerem desbastes, entretanto para consumo ao natural, o tamanho do fruto é fator limitante para algumas variedades, especialmente para as tangerinas e tangores, que por tendência à alternância de produção, caracterizada por anos de excessiva produção, seguido de ano de baixa produção, devem sofrer desbaste.
Geralmente quando a carga é excessiva, os frutos são pequenos, de baixa qualidade, coloração deficiente, aguados e ácidos, com consequente redução de preços (Pio, 1993).

Uma maneira de se contornar este problema é a realização de desbaste de frutos em anos de alta produção, amenizando o problema de alternância e melhorando a qualidade de frutos, que pode ser realizada manual ou quimicamente, onde Sharma \& Awasthi (1990) verificaram maior eficiência no desbaste químico comparado ao manual, pois as tangerinas e tangores geralmente respondem muito bem à aplicação de auxinas sintéticas e ethephon, conhecidas como reguladores vegetais que promovem abscisão (Gallash, 1988; Hutton, 1992). 
A época adequada para se proceder a aplicação de reguladores vegetais, com a finalidade de desbaste, é no período pós-florada, cerca de 30 a 40 dias após o florescimento, onde os frutinhos se encontram entre 15 a $20 \mathrm{~mm}$ de diâmetro, momento ideal para a indução da abscisão de frutos cítricos (Suzuki et al., 1990; Hutton, 1992; Ortola et al., 1998).

Segundo Guardiola (1988), a aplicação de auxinas sintéticas, como o ANA (ácido naftalenacético), estimula a abscisão de frutinhos, pois esta auxina está ligada à síntese de etileno na planta, e ambos levam a queda de frutos.

Entretanto Ortola et al.(1998) afirmam que quanto maior for o tempo para a aplicação do ANA ou ethephon, menor será a eficiência, que está intimamente relacionada com o tamanho do fruto e o período de queda natural desses frutos, que ocorre naturalmente até os $20 \mathrm{~mm}$ de diâmetro, e que o NAA, apresenta efeitos diferenciados quando aplicado em frutos cítricos. Quando aplicado em frutos com tamanho superior a $20 \mathrm{~mm}$, 0 ANA irá promover o crescimento desses frutos e não mais a abscisão, inclusive melhorando a sua fixação (Bose et al., 1988; Patil et al., 1989; Greenberg et al., 1994).

De acordo com Guan et al. (1995), a taxa de abscisão de frutos cítricos jovens está correlacionada positivamente com a atividade da celulase na zona de abscisão, pois as maiores taxas de queda coincidem com o pico de etileno, e que a alta atividade da peroxidase, com pico anterior ao do etileno na zona de abscisão, promove a abscisão dos frutos.

Como foi verificada a atuação das auxinas sintéticas e do ethephon, na síntese e no aumento dos níveis de etileno na planta, respectivamente, com a finalidade de abscisão, Galliani et al. (1975) sugeriram a aplicação de ANA e ethephon nas concentrações de 250 a $350 \mathrm{mg} \mathrm{L}^{-1}$, para desbaste de tangerinas. Sharma \& Awasthi (1988, 1990), aplicando especificamente em tangerineira 'Kinnow', ANA a $350 \mathrm{mg} \mathrm{L}^{-1}$, obtiveram maior porcentagem de abscisão do que o ethephon na dose de $200 \mathrm{mg} \mathrm{L}^{-1}$.

O presente trabalho teve por objetivo avaliar a eficiência dos reguladores vegetais, ANA e ethephon no desbaste químico de frutos de tangor 'Murcote' (Citrus reticulata Blanco x Citrus sinensis L. Osbeck), aplicados nas plantas sadias em pleno desenvolvimento dos frutos.

\section{MATERIAL E MÉTODOS}

O presente trabalho foi desenvolvido a campo, na Fazenda Triângulo, Município de Pratânia, Estado de São Paulo, nas coordenadas geográficas $22^{\circ} 44^{\prime}$ de latitude Sul e $48^{\circ} 34^{\prime}$ de longitude Oeste de Greenwich, com $750 \mathrm{~m}$ de altitude. O clima é classificado, segundo KOPPEN, como mesotérmico Cfa, que significa clima temperado chuvoso, com total de chuvas no mês mais seco menor que $40,7 \mathrm{~mm}$ e onde o mês mais quente apresenta temperatura média de $23,8^{\circ} \mathrm{C}$, e o mês mais frio de $17,5^{\circ} \mathrm{C}$, com temperatura média anual de $21^{\circ} \mathrm{C}$ e precipitação média anual de $1445 \mathrm{~mm}$.

As plantas utilizadas no experimento, foram do híbrido tangor 'Murcote' (Citrus reticulata Blanco x Citrus sinensis L. Osbeck) de 5 anos de idade, enxertadas sobre limoeiro 'Cravo' (Citrus limonia L. Osbeck), e plantadas no espaçamento 3,5 x 7,0 metros. As plantas receberam os seguintes tratamentos: testemunha, ANA (ácido naftalenacético) nas doses de 100, 200, 300 e 400 $\mathrm{mg} \mathrm{L}^{-1}$ e ethephon (etileno) nas doses de 200, 300 e 400 $\mathrm{mg} \mathrm{L}^{-1}$, em solução aquosa juntamente com espalhante adesivo não iônico a 0,05\%.

A auxina utilizada foi a de nome comercial Nafusaku, na concentração de $10 \%$ de i.a., e o ethephon, de nome comercial Ethrel, na concentração $240 \mathrm{~g} \mathrm{~L}^{-1} \mathrm{de}$ i.a., e o espalhante adesivo utilizado foi o Extravon (25\% alquil-fenol-poliglicoleter). As pulverizações foram realizadas pelo período da manhã, até as 10 horas, evitando-se assim umidade relativa do ar inferior a $50 \%$. As plantas foram pulverizadas com pulverizador costal, capacidade $20 \mathrm{~L}, 40$ dias após o pleno florescimento, com frutos apresentando $15 \mathrm{~mm}$ de diâmetro.

Foram realizadas avaliações periódicas sendo a primeira, realizada anteriormente à aplicação dos reguladores vegetais e outras avaliações 15 dias após a aplicação. $O$ delineamento utilizado foi em blocos ao acaso, três plantas por parcela, oito tratamentos e quatro repetições, totalizando 96 plantas no experimento. As avaliações foram baseadas na contagem de frutos em dois ramos previamente marcados em cada planta.

\section{RESULTADOS E DISCUSSÃO}

A aplicação de ethephon sobre plantas de tangor 'Murcote' promoveu o desbaste de frutos em todas as doses, e apesar de não existirem diferenças estatísticas entre os tratamentos com ethephon, a dose de 400 $\mathrm{mg} \mathrm{L}^{-1}$ mostrou tendência de maior porcentagem de queda de frutos, que atingiu 66,57\% (TABELA 1). Mesmo na mais alta dose, o ethephon não induziu abscisão foliar, prejudicial à planta. Dados que concordam com ElKassas et al.(1994) e Salen et al., (1995), que obtiveram queda de frutos com a aplicação de ethephon nas doses de 200 a $400 \mathrm{mg} \mathrm{L}^{-1}$, em tangerinas 'Balady' e 'Kinnow', respectivamente.

Já a aplicação de ANA, de acordo com a TABELA 1, não promoveu desbaste significativamente diferente em relação à testemunha nas doses utilizadas, provavelmente em função do tamanho dos frutos, que apesar de se encontrarem com $15 \mathrm{~mm}$, o tangor 'Murcote' apresentava uma casca mais espessa e que pode ter impedido a absorção de quantidade suficiente da auxina, responsável pela indução da síntese de etileno no fruto, e consequentemente a abscisão desses frutos. 
TABELA 1 - Porcentagem de raleio e número de frutos desbastados em plantas de tangor 'Murcote' pulverizadas com reguladores vegetais, ANA e ethephon, 40 dias após o pleno florescimento.

\begin{tabular}{lcc}
\hline Tratamento & \% de raleio & $\begin{array}{c}\text { Número de } \\
\text { frutos } \\
\text { desbastados }\end{array}$ \\
\hline 1 - testemunha & $4,95 \mathrm{c}$ & $6 \mathrm{~d}$ \\
2 - ANA $100 \mathrm{mg} \mathrm{L}^{-1}$ & $7,28 \mathrm{c}$ & $8 \mathrm{~d}$ \\
3 - ANA $200 \mathrm{mg} \mathrm{L}^{-1}$ & $14,77 \mathrm{cb}$ & $50 \mathrm{cb}$ \\
4 - ANA $300 \mathrm{mg} \mathrm{L}^{-1}$ & $6,79 \mathrm{c}$ & $17 \mathrm{dcb}$ \\
5 - ANA $400 \mathrm{mg} \mathrm{L}^{-1}$ & $23,25 \mathrm{cb}$ & $60 \mathrm{dcb}$ \\
6 - Ethrel $200 \mathrm{mg} \mathrm{L}^{-1}$ & $41,56 \mathrm{ab}$ & $120 \mathrm{ba}$ \\
7 - Ethrel $300 \mathrm{mg} \mathrm{L}^{-1}$ & $43,02 \mathrm{ab}$ & $98 \mathrm{ca}$ \\
8 - Ethrel $400 \mathrm{mg} \mathrm{L}^{-1}$ & $66,57 \mathrm{a}$ & $175 \mathrm{a}$ \\
\hline DMS (1\%) & 24,43 & 3,19 \\
CV (\%) & 37,19 & 37,90 \\
\hline
\end{tabular}

Médias seguidas de mesma letra, na vertical, não diferem significativamente pelo teste Tukey, a $5 \%$.

Além disso, Monselise (1979) relaciona o sucesso de queda de frutos com condições ambientais após a aplicação do ANA, principalmente o efeito da temperatura, onde a abscisão pode ser extremamente significativa, em temperaturas muito altas, efeito pouco significativos em temperaturas amenas.

Farmahan (1992), utilizando tangerineira 'Kinnow' (C. nobilis $x$ C. deliciosa), obteve diferenças bastante significativas no desbaste com a aplicação de ANA e ethephon, aos 31 dias após o florescimento, onde 0 ethephon promoveu abscisão de frutos da ordem de $60 \%$, e o ANA na ordem de $27 \%$, entretanto o ethephon promoveu queda de folhas da ordem de $5 \%$. Além desta diferença, o ANA mostrou-se menos efetivo na regulação de alternância de produção, concordando com Brar et al. (1992) e Ragone (1994), que também observaram diferenças siginificativas entre a aplicação de ANA e ethephon em tangerineira 'Kinnow'.

Aplicações tardias dos reguladores vegetais, prejudicam o efeito de abscisão, principalmente com o ANA, como já foi sugerido anteriormente, e confirmado por Kock et al. (1997) que observou este efeito em tangerineira 'Clementina', com o ANA e o ethephon aplicados 60 dias após o pleno florescimento, entretanto a auxina não mostrou efeito de desbaste, enquanto que o ethephon promoveu queda de frutos de $60 \%$.

Outro fator que pode interferir na eficiência do ANA na abscisão de frutos, é o maior número de folhas nos ramos reprodutivos, que acaba por reduzir o efeito do ANA na síntese de etileno (Ortola et al., 1998).

Já o efeito do ethephon é considerado mais efetivo na queda de frutos, mesmo em várias cultivares e espécies cítricas, porém em doses acima de $500 \mathrm{mg}$ $\mathrm{L}^{-1}$, pode levar a abscisão de folhas.

\section{CONCLUSÕES}

O ethephon é mais efetivo no desbaste de frutos de tangor 'Murcote', com o objetivo de melhorar a qualidade dos frutos, e também reduzir os efeitos da alternância de produção, entretanto em doses que não ultrapassem $500 \mathrm{mg} \mathrm{L}^{-1}$, evitando assim a abscisão foliar. A aplicação de ANA mostrou-se não efetiva, mas que, dependendo de características intrínsecas da planta e fatores ambientais, pode mostrar-se também efetivo no raleio de frutos.

\section{REFERÊNCIAS BIBLIOGRÁFICAS}

AGRIANUAL-2000. São Paulo: Argos comunicação, 2000. p.318329.

BOSE, T.K.; HUSSAIN, T.; MITRA, S.K.; ROY, A. Control of premature fruit drop in mandarin orange. Haryana Journal of Horticultural Science, v.17, p.140-143, 1988.

BRAR, S.S.; MINHAS, P.P.S.; KAUNDAL, G.S.; SUBHADRABANDHU, S. Chemical thinning of Kinnow mandarin. Acta Horticulture, n.321, p.443-448, 1992.

EL-KASSAS, S.E.; AHMED, M.A.; EL-SESE, A.M.; MOHAMED, A.A. Physiological studies on some factors affecting alternate bearing in Balady mandarin (Citrus reticulata Blanco). A effect of fruit thinning during on flowering season by certain growth regulators. Assiut Journal of Agricultural Sciences, v.25, p.141-153, 1994.

FARMAHAN, H.L. Chemical thinning improves 'off' year crop harvest in Kinnow mandarin. Horticultural Journal, v.5, p.6772, 1992.

GALLASH, P.T. Chemical thinning of heavy crops of mandarins to increase fruit size. In: INTERNATIONAL CITRUS CONGRESS, 6., Middle-East, 1988. Proceedings. MiddleEast, 1988. p.395-405.

GALLIANI, S.; MONSELISE, S.P.; GOREN, R. Improving fruit size and breaking alternate bearing in 'wilking' mandarins by CEPA and other agents. HortScience, n.10, p.68-69, 1975.

GREENBERG, J.; HERTZANO, Y.; ESHEL, G. Effects of 2,4-D, ethephon, and NAA on fruit size and yield of Star Rubi red grapefruit. Proceedings of the International Society of Citriculture, v.1, p.520-523, 1994.

GUAN, Y.L.; HU, A.S.; JIANG, B.F.; MO, L.H. Hormonal control on the abscission of citrus fruits. Acta Horticulture Zhegiangensis, v.7, p.297-300, 1995.

GUARDIOLA, J.L. Factors limiting productivity in citrus. A physiological approach. Proceedings of the International Society of Citriculture, v.1, p.381-394. 1988.

HUTTON, R.J. Improving fruit size and packout of late valencia oranges with ethephon fruit thinning sparys. Australian Journal of Experimental Agriculture, v.32, p.753-758, 1992.

KOCK, N.; THERON, K.I.; RABE, E. The effect of naphthalene acetic acid and ethephon application during the physiological fruit drop period on yield and fruit size of 'clementine' mandarin (Citrus reticulata blanco). Journal of the Southern African Society for Horticultural Sciences, v.7, p.55-57, 1997.

MONSELISE, S.P. The use of growth regulators in citriculture: a review. Scientia Horticulturae, v.11, p.151-162, 1979.

ORTOLA, A.G.; MONERRI, C.; GUARDIOLA, J.L.; GARCIAMARTINEZ, J.L.; QUINLAN, J.D. Fruitlet age and inflorescence characteristics affect the thinning and the increase in fruitlet growth rate induced by auxin applications in citrus. Acta Horticulturae, n.463, p.501-508, 1998. 
PATIL, V.S.; KEDAR, V.P.; NAGRE, P.K. Effect of foliar sprays of plant growth regulators and urea on premature and preharvest fruit drop in Kinnow mandarin. PKV Research Journal, v.13, p.161-163, 1989.

PIO, R.M. Tangerinas para o verão. Laranja, v.14, p.539-549, 1993.

RAGONE, M.L. Effect of growth regulators and cultural practices on the production and quality of satsumas mandarins. Revista de Investigaciones Agropecuárias, v.25, p.57-63, 1994.

SALEN, S.E.; GUINDY, L.F.; IBRAHIEM, T. A. Possibility of regulating alternate bearing in Balady mandarin trees by naphthalene acetic acid. Bulletin of the Faculty of the University of Cairo, v.46, p.253-263, 1995.
SHARMA, R.K.; AWASTHI, R.P. Effect of growth regulators on crop regulation in Kinnow. Punjab Horticultural Journal, v.28, p.22-26, 1988.

SHARMA, R.K.; AWASTHI, R.P. Effects of growth regulators on crop regulation of Kinnow (Citrus nobilis $x$ citrus deliciosa). Indian Journal of Horticulture, v.47, p.162-166, 1990.

SUZUKI, K.; KAWASE, K.; HIRAI, K. The effects of mixing ethephon with ethylchlosate on fruit thinning and defoliation in satsuma mandarin (Citrus unshiu Marcovitch). Bulletin of the Fruit Tree Research Station, n.17, p.45-54, 1990.

$\overline{\text { Recebido em } 05.06 .00}$ 